\title{
VISCOSITY SOLUTIONS ASSOCIATED WITH IMPULSE CONTROL PROBLEMS FOR PIECEWISE-DETERMINISTIC PROCESSES
}

SUZANNE M. LENHART

\author{
UNIVERSITY OF TENNESSEE \\ Mathematics DePaRTMENT \\ KNOXVILle, TenNessee 37996-1300
}

(Received July 1, 1987 and in revised form Janurary 5, 1988)

ABSTRACT. This paper considers existence and uniqueness results for viscosity solutions of integro-differential equations associated with the impulse control problem for piecewisedeterministic processes on bounded domains and on $\mathbf{R}^{\mathbf{n}}$.

KEY WORDS AND PHRASES. Impulse control, viscosity solution, piecewise-deterministic processes.

1980 AMS SUBJECT CLASSIFICATION CODE. 35F20

\section{INTRODUCTION.}

This paper considers viscosity solutions of integro-differential equations associated with the impulse control problem for piecewise-deterministic (PD) processes

$$
\max (L u-f, u-M u)=0 \text { in } E
$$

where

$$
\begin{aligned}
L u(x) & =-\sum_{i=1}^{n} g_{i}(x) u_{x_{i}}(x)+\alpha(x) u(x)-\lambda(x) \int_{E}(u(y)-u(x)) Q(d y, x) \\
M u(x) & =\inf _{\substack{x+\xi \bar{E} \\
\xi \geq 0}}(u(x+\xi)+c(\xi)) .
\end{aligned}
$$


We consider the cases when $E=\Omega$, a bounded domain in $\mathbf{R}^{n}$, and $E=\mathbf{R}^{n}$. In the bounded domain case, we have the following boundary condition:

$$
u(x)=\int_{\Omega} u(y) Q(d y, x) \text { for all } x \epsilon \partial \Omega
$$

Let us briefly give the background for this problem. A PD process, $x(t)$, with jump rate, $\lambda(x)$, and jump distribution $Q(d y, x)$, follows deterministic dynamics between random jumps:

$$
\frac{d x(t)}{d t}=\left(g_{1}(x(t)), \ldots, g_{n}(x(t))\right)
$$

Davis [1] developed the probabilistic side of these PD Markov processes. If the $i$ th jump of the process occurs at $T_{i}$, then the distribution of $x\left(T_{i}\right)$ is $Q\left(d y, x\left(T_{i}^{-}\right)\right)$and

$$
P\left(T_{i+1}-T_{i}>t\right)=\exp \left(-\int_{0}^{t} \lambda\left(x\left(T_{i}+s\right)\right) d s\right) .
$$

Davis [1] showed that a PD process is a strong Markov process with generator

$$
\sum_{i=1}^{n} g_{i}(x) u_{x_{i}}+\lambda(x) \int_{E}(u(y)-u(x)) Q(d y, x),
$$

with $E$, the state space. The boundary condition occurs because the PD process jumps back into the interior of $\Omega$, upon hitting the boundary of $\Omega$. The jumps $T_{i}$ are part of the process, $x(t)$. Consider when certain jumps, "impulses", are controlled from outside the PD process. Suppose the state is changed from $x$ to $x+\xi$ with impulse $\xi \geq 0$, and cost $\ell(\xi)$ is incurred when the impulse $\xi$ is applied. An impulse control strategy $v$ is a sequence of stopping times and impulses,

$$
v=\left\{\theta_{1}, \xi_{1}, \theta_{2}, \xi_{2}, \ldots\right\}, \quad\left(\theta_{i} \rightarrow \infty \text { as } i \rightarrow \infty\right) .
$$

The controlled PD process $x^{v}$ satisfies

$$
x^{v}\left(\theta_{i}+0\right)=x^{v}\left(\theta_{i}-0\right)+\xi_{i}
$$

The associated cost function is

$$
J_{x}(v)=E_{x}\left[\int_{0}^{\infty} f\left(x^{v}(t)\right) e^{-\alpha t} d t+\sum_{i=1}^{\infty} c\left(\xi_{i}\right) e^{-\alpha \theta_{1}}\right]
$$

The minimal cost function is

$$
V(x)=\inf _{v} J_{x}(v)
$$

Heuristically, the dynamic programming equation satisfied by the minimal cost function is given by (1.1).

For results on optimal control of PD processes, see Davis [2], Vermes [3], Soner [4], Lenhart and Liao [5, 6], and Gugerli [7]. See Barles [8] for deterministic impulse control. 
In this paper, we define the notion of viscosity solution of (1.1) and prove existence and uniqueness results in the viscosity sense. The control representation is discussed in the last section.

\section{UNIQUENESS.}

The original formulations of viscosity solution definitions, by Crandall and Lions $[9,10]$, did not include integral terms in the operators, so we state the definition extension to this case.

DEFINITION: $u \in B U C(\bar{E})$ (bounded, uniformly continuous) ( $E$ will be $\Omega$ or $\mathbf{R}^{n}$ )

(i) $u$ is a viscosity subsolution of (1.1) if

$$
\begin{aligned}
& \max \left(-g_{i}\left(x_{0}\right) \phi_{x_{1}}\left(x_{0}\right)+\alpha\left(x_{0}\right) u\left(x_{0}\right)\right. \\
& -\lambda\left(x_{0}\right) \int_{E}\left(u(y)-u\left(x_{0}\right)\right) Q\left(d y, x_{0}\right)-f\left(x_{0}\right), \\
& \left.u\left(x_{0}\right)-M u\left(x_{0}\right)\right) \leq 0
\end{aligned}
$$

whenever $\phi \epsilon C^{1}(E)$ and $u-\phi$ has a global maximum at $x_{0}$.

(ii) $u$ is a viscosity supersolution of (1.1) if

$$
\begin{aligned}
& \max \left(-g_{i}\left(x_{0}\right) \phi_{x_{i}}\left(x_{0}\right)+\alpha\left(x_{0}\right) u\left(x_{0}\right)\right. \\
& -\lambda\left(x_{0}\right) \int_{E}\left(u(y)-u\left(x_{0}\right)\right) Q\left(d y, x_{0}\right)-f\left(x_{0}\right), \\
& \left.u\left(x_{0}\right)-M u\left(x_{0}\right)\right) \geq 0
\end{aligned}
$$

whenever $\phi \epsilon C^{1}(E)$ and $u-\phi$ has a global minimum at $x_{0}$.

Note that implicit summation on repeated subscripts is used on the $g_{2}$ terms above.

We make the following assumptions:

$\lambda, f, \alpha$ bounded uniformly continuous on $E$,

$g_{i}$ Lipschitz continuous on $E, i=1, \ldots, n$,

$\alpha(x) \geq \alpha_{0}>0$ on $E$,

$\lambda(x) \geq 0, f(x) \geq 0$ on $E$,

for each fixed $x \epsilon E, Q(d y, x)$ is a probability measure which is Lipschitz continuous as a function of $x$, i.e.,

$$
\left|\int_{E} \phi(y) Q(d y, x)-\int_{E} \phi(y) Q(d y, z)\right| \leq C_{Q}\|\phi\|_{L^{\infty}}|x-z| \text { for all } \phi \epsilon L^{\infty}(E),
$$

and $c(\cdot)$ is a continuous, subadditive, increasing, positive function on $\left(\mathbf{R}^{+}\right)^{n}$ with 


$$
\begin{aligned}
& c(\xi) \rightarrow \infty \text { as } \xi \rightarrow \infty, \\
& c(0)=k, \\
& c(\xi) \geq k>0 \text { for all } \xi \geq 0 .
\end{aligned}
$$

We assume that $\Omega$ is a smooth bounded domain in $\mathbf{R}^{n}$.

We need an assumption that guarantees

$$
u \epsilon C(\bar{\Omega}) \Rightarrow M u \epsilon C(\bar{\Omega})
$$

from Lions and Perthame [11], we have the needed assumption:

$$
\begin{aligned}
& \text { for all } x \epsilon \bar{\Omega}, \quad\{\xi \geq 0 \mid \xi \neq 0, x+\xi \epsilon \partial \Omega, \exists \varepsilon>0 \text {, s.t. } \forall y \geq 0, \\
& x+y \notin \Omega \text { if }|y-\xi| \leq \varepsilon\} \text { is empty. }
\end{aligned}
$$

If $\Omega$ is convex, then $(2.10)$ holds.

We now prove comparison results in $\Omega$ and then in $\mathbf{R}^{n}$ that yield uniqueness results for equation (1.1).

THEOREM 2.1. Under assumptions (2.3)-(2.10) on $\Omega$, if $u$ is a viscosity subsolution of (1.1) on $\Omega$ and $v$ is a viscosity supersolution of (1.1) on $\Omega$ with

$$
u(x) \leq \int_{\Omega} u(y) Q(d y, x) \text { and } v(x) \geq \int_{\Omega} v(y) Q(d y, x) \text { for all } x \in \partial \Omega,
$$

then $u \leq v$ on $\bar{\Omega}$.

Proof: Let $0<\mu<1$ and set $w=\mu u$. There exists $z$ in $\bar{\Omega}$ such that

$$
(w-v)(z)=\max _{x \in \bar{\Omega}}(w-v)(x) .
$$

First we show that we can choose $z$ so that $z \notin \partial \Omega$. If $z \in \partial \Omega$, then

$$
\begin{aligned}
\max (w-v)=w(z)-v(z) & \leq \int_{\Omega}(w(y)-v(y)) Q(d y, z) \text { by }(2.11) \\
& \leq \sup (w-v) .
\end{aligned}
$$

If the maximum of $w-v$ does not occur at an interior point, then there exists a set $A \subset \Omega$ such that

$$
Q(A, z)>0 \text { and } w(y)-v(y)<\max (w-v) \text { for all } y \epsilon A
$$

Then

$$
\int_{\Omega}(w(y)-v(y)) Q(d y, z)<\sup (w-v),
$$

which is a contradiction. So there exists $\bar{z} \in \Omega$ where the maximum occurs. Thus we can assume $z \in \Omega$. 
Set $M=\max \left(\|u\|_{\infty},\|v\|_{\infty}\right)$. Define

$$
\Phi_{\varepsilon}(x, y)=w(x)-v(y)-\left|\frac{x-y}{\varepsilon}\right|^{2}-C_{\varepsilon}|y-z|^{2}
$$

where $C_{\varepsilon}=\sqrt{\omega_{v}(\sqrt{2 M} \varepsilon)}$ and $\omega_{v}$ is a modulus of continuity for $v$. There exists $\left(x^{e}, y^{e}\right) \in \bar{\Omega}$ such that

Then

$$
\sup _{x, y \in \bar{\Omega}} \Phi_{e}(x, y)=\Phi_{e}\left(x^{e}, y^{e}\right)
$$

$$
\Phi_{e}(z, z)=w(z)-v(z) \leq \Phi_{e}\left(x^{e}, y^{e}\right)
$$

implies

$$
\begin{aligned}
\left|\frac{x^{e}-y^{e}}{\varepsilon}\right|^{2}+C_{\varepsilon}\left|y^{e}-z\right|^{2} & \leq w\left(x^{\varepsilon}\right)-v\left(x^{e}\right)-(w(z)-v(z))+v\left(x^{\varepsilon}\right)-v\left(y^{\varepsilon}\right) \\
& \leq 2 M .
\end{aligned}
$$

Refining this estimate gives

$$
\left|x^{e}-y^{\varepsilon}\right| \leq \sqrt{2 M} \varepsilon
$$

and then

$$
\begin{aligned}
\left|\frac{x^{e}-y^{e}}{\varepsilon}\right|^{2}+C_{e}\left|y^{e}-z\right|^{2} & \leq \omega_{v}\left(\left|x^{e}-y^{e}\right|\right) \\
& \leq \omega_{v}(\sqrt{2 M} \varepsilon)
\end{aligned}
$$

This implies

$$
\left|\frac{x^{\varepsilon}-y^{\varepsilon}}{\varepsilon}\right| \rightarrow 0 \text { as } \varepsilon \rightarrow 0
$$

Also

$$
C_{e}\left|y^{\varepsilon}-z\right|^{2} \leq C_{\varepsilon}^{2}
$$

and then

$$
\left|y^{\epsilon}-z\right| \rightarrow 0 \text { as } \varepsilon \rightarrow 0
$$

Notice also we have for $\varepsilon$ sufficiently small, $x^{\varepsilon}, y^{\varepsilon} \in \Omega$ since $z \in \Omega$.

Since $w$ is a viscosity subsolution of

$$
\begin{gathered}
\max \left(-g_{i} w_{x_{i}}+\alpha w-\lambda \int_{\Omega}(w(y)-w(x)) Q(d y, x)-\mu f, w-\widetilde{M} w\right)=0, \\
\widetilde{M} w(x)=\inf _{\substack{x+\xi \in \bar{\Omega} \\
\xi \geq 0}}(w(x+\xi)+\mu C(\xi))
\end{gathered}
$$

and $x \rightarrow w(x)-\left(v(y)-\left|\frac{x-y^{e}}{e}\right|^{2}-C_{\varepsilon}\left|y^{\varepsilon}-z\right|^{2}\right)$ has a maximum at $x^{\varepsilon}$, 


$$
\begin{array}{r}
\max \left(-g_{\mathrm{v}}\left(x^{\varepsilon}\right)\left(2\left(\frac{x^{\varepsilon}-y^{\varepsilon}}{\varepsilon^{2}}\right)_{i}\right)\right. \\
+\alpha\left(x^{\varepsilon}\right) w\left(x^{\varepsilon}\right)-\lambda\left(x^{\varepsilon}\right) \int_{\Omega}\left(w(y)-w\left(x^{\epsilon}\right)\right) Q\left(d y, x^{\varepsilon}\right)-\mu f\left(x^{\epsilon}\right), \\
\left.w\left(x^{\varepsilon}\right)-\widetilde{M} w\left(x^{\epsilon}\right)\right) \leq 0 .
\end{array}
$$

Since $v$ is a supersolution of (1.1) and

$$
y \rightarrow v(y)-\left(w\left(x_{0}\right)-\left|\frac{x_{0}-y}{\varepsilon}\right|^{2}-C_{\varepsilon}\left|y_{0}-z\right|^{2}\right),
$$

has a minimum at $y$,

$$
\begin{aligned}
& \max \left(-g_{2}\left(y^{e}\right)\right.\left(2\left(\frac{x^{e}-y^{e}}{\varepsilon^{2}}\right)+2 C_{\varepsilon}\left(y^{\varepsilon}-z\right)_{i}\right) \\
&+\alpha\left(y^{e}\right) v\left(y^{e}\right)-\lambda\left(y^{e}\right) \int_{\Omega}\left(v(y)-v\left(y^{e}\right)\right) Q\left(d y, y^{e}\right)-f\left(y^{e}\right) \\
&\left.v\left(y^{e}\right)-M v\left(y^{e}\right)\right) \geq 0 .
\end{aligned}
$$

CASE A. $v\left(y^{e}\right) \geq M v\left(y^{e}\right)$.

There exists $\xi^{1} \geq 0$ such that $M v\left(x^{e}\right)=v\left(x^{e}+\xi^{1}\right)+c\left(\xi^{1}\right)$. From property (2.10), $M v \in C(\bar{\Omega})$, and $M v\left(x^{\varepsilon}\right)-M v\left(y^{\varepsilon}\right)$ can be made small for $\varepsilon$ small.

$$
\begin{aligned}
\Phi_{e}\left(x^{e}, y^{e}\right) \leq & w\left(x^{e}\right)-M v\left(y^{\varepsilon}\right)-\left|\frac{x^{e}-y^{e}}{\varepsilon}\right|^{2}-C_{e}\left|y^{e}-z\right|^{2} \\
\leq & w\left(x^{e}\right)-M v\left(x^{e}\right)-\left|\frac{x^{e}-y^{e}}{\varepsilon}\right|^{2}-C_{\varepsilon}\left|y^{e}-z\right|^{2}+M v\left(x^{e}\right)-M v\left(y^{e}\right) \\
\leq & w\left(x^{e}+\xi^{1}\right)+\mu c\left(\xi^{1}\right)-\left(v\left(x^{\varepsilon}+\xi^{1}\right)+c\left(\xi^{1}\right)\right) \\
& -\left|\frac{x^{e}-y^{e}}{\varepsilon}\right|^{2}-C_{\varepsilon}\left|y^{e}-z\right|^{2}+M v\left(x^{e}\right)-M v\left(y^{e}\right) .
\end{aligned}
$$

Using $(\mu-1) c\left(\xi^{1}\right) \leq(\mu-1) k$,

$$
\begin{aligned}
\Phi_{e}\left(x^{e} y^{e}\right) \leq w & \left(x^{e}+\xi^{1}\right)-v\left(x^{\varepsilon}+\xi^{1}\right)-C_{\varepsilon}\left|x^{\varepsilon}+\xi^{1}-z\right|^{2} \\
& +C_{\varepsilon}\left(\left|x^{e}+\xi^{1}-z\right|^{2}-\left|y^{\varepsilon}-z\right|^{2}\right)+(\mu-1) k \\
& +M v\left(x^{\varepsilon}\right)-M v\left(y^{e}\right) .
\end{aligned}
$$

Hence for $\varepsilon$ small enough, using $(\mu-1) k<0$,

$$
\Phi_{\varepsilon}\left(x^{\varepsilon}, y^{\varepsilon}\right)<\Phi_{\varepsilon}\left(x^{\varepsilon}+\xi^{1}, x^{\varepsilon}+\xi^{1}\right)
$$

which contradicts our choice of $\left(x^{\varepsilon}, y^{\varepsilon}\right)$.

Notice this part is where $C_{\varepsilon}$ is used.

CASE B: $v\left(y^{e}\right)<M v\left(y^{e}\right)$.

Using (2.14) and (2.15), 


$$
\begin{aligned}
& \alpha(w(z)-v(z)) \leq \alpha\left(x^{\varepsilon}\right)\left(w\left(x^{\varepsilon}\right)-v\left(x^{\varepsilon}\right)\right)+o_{\varepsilon}(1)+\alpha\left(y^{\varepsilon}\right) v\left(y^{\varepsilon}\right)-\alpha\left(y^{\varepsilon}\right) v\left(y^{\varepsilon}\right) \\
& \leq o_{\varepsilon}(1)+\alpha\left(x^{\varepsilon}\right) w\left(x^{e}\right)-\alpha\left(y^{e}\right) v\left(y^{e}\right) \\
& \leq-2\left(g_{\mathrm{v}}\left(y^{\varepsilon}\right)-g_{\mathrm{v}}\left(x^{e}\right)\right)\left(\frac{x^{e}-y^{e}}{\varepsilon^{2}}\right)+f\left(y^{e}\right)-\mu f\left(x^{e}\right) \\
& -2 g_{\mathrm{i}}\left(y^{\varepsilon}\right) C_{e}\left(y^{\varepsilon}-z\right)_{i}-\lambda\left(y^{\varepsilon}\right) \int_{\Omega}\left(v(y)-v\left(y^{\varepsilon}\right)\right) Q\left(d y, y^{\varepsilon}\right) \\
& +\lambda\left(x^{e}\right) \int_{\Omega}\left(w(y)-w\left(x^{\varepsilon}\right)\right) Q\left(d y, x^{e}\right) \\
& \leq C_{f}(1-\mu)+o_{\varepsilon}(1) \text {. }
\end{aligned}
$$

(Note that $o_{\varepsilon}(1) \rightarrow 0$ as $\varepsilon \rightarrow 0$.) See Lenhart [12] for an estimate on such integral terms; the key idea used is that

$$
\int_{\Omega}[(w(y)-v(y))-(w(z)-v(z))] Q\left(d y, x^{e}\right) \leq 0
$$

and the integral terms in (2.16) are close to the integral terms in (2.17). Thus we conclude

$$
\max _{x}(w(x)-v(x)) \leq C(1-\mu)+o_{\varepsilon}(1)
$$

Let $\mu \rightarrow 1$,

$$
\max _{x}(u(x)-v(x)) \leq o_{\varepsilon}(1)
$$

Then let $\varepsilon \rightarrow 0$,

$$
\max (u(x)-v(x)) \leq 0
$$

Now we obtain a similar result in $\mathbf{R}^{\mathbf{n}}$.

THEOREM 2.2. Under assumptions (2.3)-(2.9) on $\mathbf{R}^{n}$, if $u$ is a viscosity subsolution of (1.1) on $\mathbf{R}^{n}$ and $v$ is a viscosity supersolution of (1.1) on $\mathbf{R}^{n}$, then

$$
u(x) \leq v(x) \text { on } \mathbf{R}^{n}
$$

Proof: Let $o<\delta<1$. Using notation from proof of Theorem 2.1, choose $z$ such that

$$
w(z)-v(z) \geq \sup _{x}(w-v)(x)-\delta .
$$

If $C_{\varepsilon}|y-z|^{2}+\left|\frac{x-y}{\varepsilon}\right|^{2} \geq 5 \max \left(\|u\|_{\infty},\|v\|_{\infty}\right)$, with $C_{\varepsilon}=\sqrt{\omega_{v}(\sqrt{2 M+1} \varepsilon)}$,

$$
\Phi_{\epsilon}(x, y) \leq \Phi_{\epsilon}(z, z)
$$

$\left(x^{\varepsilon \delta}, y^{\varepsilon \delta}\right)$. Thus $\Phi_{\varepsilon}$ does achieve its maximum at a finite point, say at $\left(x^{\varepsilon}, y^{\varepsilon}\right)=\left(x^{\varepsilon \delta}, y^{\varepsilon \delta}\right)$.

We obtain (2.12)-(2.15) as before, with $x^{\varepsilon \delta}-y^{\varepsilon \delta} \rightarrow 0$ as $\varepsilon \rightarrow 0$ and $\delta \rightarrow 0$.

CASE A: $v\left(y^{\varepsilon}\right) \geq M v\left(y^{\varepsilon}\right)$. By (2.8), there exists $\xi^{1} \geq 0$ such that 


$$
v\left(y^{\varepsilon}\right)>v\left(y^{e}+\xi^{1}\right)+c\left(\xi^{1}\right)-\frac{k(1-\mu)}{2}
$$

and $\left|\xi^{1}\right| \leq C_{1}$ where $C_{1}$ depends on $\|v\|_{\infty}$ but not on $\varepsilon, \delta$. Now

$$
\begin{aligned}
\Phi_{e}\left(x^{e}, y^{e}\right) \leq & w\left(x^{e}\right)-v\left(y^{e}\right)-\left|\frac{x^{\varepsilon}-y^{e}}{\varepsilon}\right|^{2}-C_{e}\left|y^{e}-z\right|^{2} \\
\leq & w\left(x^{e}+\xi^{1}\right)+\mu c\left(\xi^{1}\right)-v\left(y^{e}+\xi^{1}\right)-c\left(\xi^{1}\right)+\frac{k(1-\mu)}{2} \\
& \quad-\left|\frac{x^{e}-y^{e}}{\varepsilon}\right|^{2}-C_{e}\left|y^{e}-z\right|^{2} \\
\leq & w\left(x^{e}+\xi^{1}\right)-v\left(y^{e}+\xi^{1}\right)-\left|\frac{x^{e}-y^{e}}{\varepsilon}\right|^{2}-C_{e}\left|y^{e}+\xi^{1}-z\right|^{2} \\
& \quad+C_{e}\left(\left|y^{e}+\xi^{1}-z\right|^{2}-\left|y^{e}-z\right|^{2}\right)+\frac{k}{2}(\mu-1) \\
\leq & \Phi_{e}\left(x^{e}+\xi^{1}, y^{e}+\xi^{1}\right)+o_{e}(1)+\frac{k}{2}(\mu-1) \\
< & \Phi_{e}\left(x^{e}+\xi^{1}, y^{e}+\xi^{1}\right) \text { for } \varepsilon \text { sufficiently small. }
\end{aligned}
$$

This contradicts the choice of $\left(x^{\varepsilon}, y^{e}\right)$.

CASE B: $v\left(y^{e}\right)<M v\left(y^{e}\right)$ follows as in Theorem 2.1.

\section{EXISTENCE RESULTS.}

Due to the possible incompatibility of the impulse obstacle and boundary condition (1.2), we shall prove existence of viscosity solutions to

$$
\max (L u-f, u-M u)=0 \text { in } \Omega
$$

satisfying the boundary condition:

$$
u(x)=M u(x) \wedge \int_{\Omega} u(y) Q(d y, x) \text { for } x \epsilon \partial \Omega(\wedge \text { minimum symbol }) .
$$

Condition (3.2) formally means the state process could jump back into the interior of $\Omega$ upon hitting $\partial \Omega$ or an impulse could be used to change the state. We have the following existence result.

THEOREM 3.1. Under assumptions (2.3)-(2.10) on $\Omega$, there is a unique viscosity solution of (3.1) on $\Omega$ satisfying (3.2).

ProOF: For $\Psi \epsilon W^{1, \infty}(\bar{\Omega})$, by an extension of $[5,12]$, we have the existence of unique viscosity solution of 


$$
\max (L u-f, u-\psi)=0 \text { on } \Omega
$$

satisfying

$$
u(x)=\psi(x) \wedge \int_{\Omega} u(y) Q(d y, x) \text { for } x \epsilon \partial \Omega
$$

We also have the continuous dependence of $u$ on $\psi$, i.e., if $u_{1}, u_{2}$ are viscosity solutions of (3.3) satisfying (3.4) with obstacles $\psi_{1}, \psi_{2}$, respectively,

$$
\left\|u_{1}-u_{2}\right\|_{\infty} \leq\left\|\psi_{1}-\psi_{2}\right\|_{\infty}
$$

Using this continuous dependence result, we obtain the existence and uniqueness of viscosity solutions of (3.3) satisfying (3.4), for obstacles $\psi$ in $C(\bar{\Omega})$. Now we will apply this result with $\psi=M u$, with $u \epsilon C(\bar{\Omega})$ giving $M u \epsilon C(\bar{\Omega})$ by $(2.10)$.

We now construct a sequence which will converge to the solution of (3.1) satisfying (3.2). Define $u_{0}$ to be the unique viscosity solution of

$$
\begin{aligned}
L u_{0} & =f \text { in } \Omega \\
u_{0}(x) & =\int_{\Omega} u_{0}(y) Q(d y, x) \text { on } \partial \Omega .
\end{aligned}
$$

By (2.10), $u_{0} \epsilon C(\bar{\Omega})$ implies $M u_{0} \epsilon C(\bar{\Omega})$. Thus there exists a sequence of viscosity solutions, $\left\{u_{n}\right\}_{n=1}^{\infty}$, satisfying

$$
\begin{aligned}
& \max \left(L u_{n}-f, u_{n}-M u_{n-1}\right)=0 \text { in } \Omega \\
& u_{n}(x)=M u_{n-1}(x) \wedge \int_{\Omega} u_{n}(y) Q(d y, x) \text { on } \partial \Omega
\end{aligned}
$$

By a maximum principle argument on these viscosity solutions, we obtain

$$
0 \leq u_{n} \leq u_{n-1}, n=1,2, \ldots
$$

To get uniform convergence of our sequence, first define a map

$$
\begin{aligned}
& \sigma: C(\bar{\Omega}) \rightarrow C(\bar{\Omega}) \text { by } \\
& \sigma(\psi)=u \text { where }
\end{aligned}
$$

$u$ is the viscosity solution of (3.3) satisfying (3.4).

The map $T=\sigma \circ M$ is increasing and concave. We have the following properties for $T$ :

There exists $\bar{\lambda}$ in $(0,1)$ such that $\bar{\lambda} u_{0} \leq k$. 
If there exists $\beta \epsilon[0,1]$ such that $v-w \leq \beta w$, then $T v-T w \leq(1-\lambda) \beta T v$.

See Hanouzet and Joly [13], Perthame $[14,15]$ and Barles $[8,16]$ for examples of this tecinique. Since $u_{0}-u_{1}=u_{0}$, we iterate (j.5) to obtain

$$
u_{n-1}-u_{n} \leq(1-\bar{\lambda})^{n-1} u_{0}
$$

In this way, we obtain uniform convergence, $u_{n} \rightarrow u$. The uniform convergence of $u_{n}$ insures the convergence of the integral terms and $M u_{n-1}$, and we have that $u$ satisfies boundary condition (3.2).

To show the limit function $u$ is a viscosity solution, we first look at point $x_{0}$ where $u-\phi$ has a global minimum. Then there is a sequence $\left\{x_{n}\right\}$ converging to $x_{0}$ such that $u_{n}-\phi$ has its minimum at $x_{n}$. Since $u_{n} \leq M u_{n-1}$ on $\Omega$, we know

$$
u \leq M u \text { on } \Omega \text {. }
$$

If $u=M u$ at $x_{0}$, then the viscosity supersolution condition (2.2) is satisfied. If $u<M u$ at $x_{0}$, then $u_{n}\left(x_{n}\right)<M u_{n-1}\left(x_{n}\right)$ for $n$ large enough, which implies

$$
-g_{i} \phi_{x_{i}}+\alpha u_{n}-\lambda \int_{\Omega}\left(u_{n}(y)-u_{n}\left(x_{n}\right)\right) Q\left(d y, x_{n}\right) \geq f \text { at } x_{n}
$$

Letting $n \rightarrow \infty$, we have $u$ is a viscosity supersolution. The subsolution case follows similarly.

The uniqueness result follows as in Theorem 2.1 except for the case when the maximum of $w-v$ occurs at $z \epsilon \partial \Omega$. If

$$
v(z) \geq \int_{\Omega} v(y) Q(d y, x)
$$

then the argument goes as before. If $v(z) \geq M v(z)$, then there exists nonzero $\xi^{1} \geq 0$ such that

$$
M v(z)=v\left(z+\xi^{1}\right)+c\left(\xi^{1}\right)
$$

Then

$$
\begin{aligned}
w(z)-v(z) & \leq w\left(z+\xi^{1}\right)+\mu c\left(\xi^{1}\right)-v\left(z+\xi^{1}\right)-c\left(\xi^{1}\right) \\
& \leq w\left(z+\xi^{1}\right)-v\left(z+\xi^{1}\right)+(\mu-1) k \\
& <w\left(z+\xi^{1}\right)-v\left(z+\xi^{1}\right)
\end{aligned}
$$

which is a contradiction of the choice of $z$. 
The existence proof for the $\mathbf{R}^{n}$ case is simpler.

THEOREM 3.2. Under assumptions (2.3)-(2.9) on $\mathbf{R}^{n}$, there exists a unique viscosity solution to (1.1) in $\mathbf{R}^{n}$.

FroOF: We use the iterative approximation scheme on $\mathbf{R}^{\boldsymbol{n}}$,

$$
\max \left(L u_{n}-f, u_{n}-M u_{n-1}\right)=0 \text { in } \mathbf{R}^{n}, n=1,2, \ldots \text { and } L u_{0}=f \text { in } \mathbf{R}^{n}
$$

To obtain existence for (3.6), we use extensions of results from $[5,12]$ with $\psi$ in $B U C\left(\mathbf{R}^{n}\right)$. Note that $u_{n-1} \epsilon B U C\left(\mathbf{R}^{n}\right)$ implies $M u_{n-1} \epsilon B U C\left(\mathbf{R}^{n}\right)$. By using an operator $T$ as in Theorem 3.1, we obtain uniform convergence of $\left\{u^{n}\right\}$ to a viscosity solution $u$. The uniqueness result is Theorem 2.2 .

\section{CONTROL REPRESENTATION.}

To put the results obtained in section 3 in the context of the classical results on impulse control (Bensoussan and Lions [17] and Menaldi [18]) we show that the solution obtained in Theorem 3.2 is equal to the value function associated with the impulse control problem (1.4).

THEOREM 4.1. The unique viscosity solution $u(x)$ from Theorem 3.2 is equal to the value function $V(x)$ from (1.4)

ProOF: First, we show that the approximations $u_{n}$ have the following control interpretation:

$$
u_{n}(x)=\inf \left\{J_{x}\left(v_{n}\right): v_{n} \text { impulse control strategy with } \theta_{i}=\infty, \text { for all } i \geq n+1\right\}
$$

i.e., $u_{n}$ is the minimum cost function associated with the impulse control problem with at most $n$ impulses allowed. We show (4.1) by induction. Call the right hand side of (4.1), $V_{n}(x)$. The representation for $u_{0}$ (no impulses) is valid. Assume $(4.1)$ for $u_{n-1}$. By $[5,12]$, we have

$$
u_{n}(x)=\inf _{\theta} E_{x}\left[\int_{0}^{\theta} f(x(t)) e^{-\alpha t} d t+M u_{n-1}(x(\theta)) e^{-\alpha \theta}\right]
$$

where $\theta$ is a stopping time. Let $v_{n}$ be an impulse control strategy with $n$ impulses,

$$
v_{n}=\left(\theta_{1}, \xi_{1}, \ldots, \theta_{n}, \xi_{n}\right)
$$

By (4.2), and writing $x_{n}(t)=x^{v_{n}}(t)$ from (1.3), 


$$
\begin{aligned}
u_{n}(x) & \leq E_{x}\left[\int_{0}^{\theta_{1}} f\left(x_{n}(t)\right) e^{-\alpha t} d t+M u_{n-1}\left(x_{n}\left(\theta_{1}-0\right)\right) e^{-\alpha \theta_{1}}\right] \\
& \leq E_{x}\left[\int_{0}^{\theta_{1}} f\left(x_{n}(t)\right) e^{-\alpha t} d t+e^{-\alpha \theta_{1}}\left(u_{n-1}\left(x_{n}\left(\theta_{1}-0\right)+\xi_{1}\right)+c\left(\xi_{1}\right)\right)\right] \\
& \leq E_{x}\left[\int_{0}^{\theta_{1}} f\left(x_{n}(t)\right) e^{-\alpha t} d t+e^{-\alpha \theta_{1}} c\left(\xi_{1}\right) \quad \text { (use (4.1) on } u_{n-1}\right) \\
& \left.+e^{-\alpha \theta_{1}} E_{x_{n}\left(\theta_{1}\right)+\xi_{1}}\left(\int_{\theta_{1}}^{\infty} f\left(x_{n}(t)\right) e^{-\alpha\left(t-\theta_{1}\right)} d t+\sum_{i=2}^{n} e^{-\alpha\left(\theta_{i}-\theta_{1}\right)} c\left(\xi_{i}\right)\right)\right]
\end{aligned}
$$

Therefore

$$
u_{n}(x) \leq V_{n}(x)
$$

To show $u_{n} \geq V_{n}$, for $\varepsilon>0$, choose $\theta_{1}$ such that

$$
E_{x} \int_{0}^{\theta_{1}} f(x(t)) e^{-\alpha t} d t+e^{-\alpha \theta_{1}} M u_{n-1}\left(x\left(\theta_{1}-0\right)\right)<u_{n}(x)+\varepsilon / 2
$$

Using (2.8), choose $\xi_{1}$ such that

$$
M u_{n-1}\left(x\left(\theta_{1}-0\right)\right)=u_{n-1}\left(x\left(\theta_{1}-0\right)+\xi_{1}\right)+c\left(\xi_{1}\right)
$$

By the inductive hypothesis on $u_{n-1}\left(x\left(\theta_{1}-0\right)+\xi_{1}\right)$, there exists an impulse control strategy

$$
v_{n-1}=\left(\theta_{2}, \xi_{2}, \ldots, \theta_{n}, \xi_{n}\right), \quad \theta_{2}>\theta_{1},
$$

such that

$$
J_{x\left(0_{1}\right)+\xi_{1}}\left(v_{n-1}\right)<u_{n-1}\left(x\left(\theta_{1}-0\right)+\xi_{1}\right)+\varepsilon / 2
$$

By (4.3)-(4.5),

$$
\begin{aligned}
& E_{z}\left[\int_{0}^{\theta_{1}} f(x(t)) e^{-\alpha t} d t+e^{-\alpha \theta_{1}} E_{z\left(\theta_{1}\right)+\xi_{1}}\left[\int_{0_{1}}^{\infty} f\left(x_{n-1}(t)\right) e^{-\alpha\left(t-\theta_{1}\right)} d t\right.\right. \\
& \left.\left.+\sum_{i=1}^{n} e^{-\alpha\left(\theta_{i}-\theta_{1}\right)} c\left(\xi_{i}\right)-\varepsilon / 2\right]\right]<u_{n}(x)+\varepsilon / 2
\end{aligned}
$$

which implies

$$
V_{n}(x) \leq J_{x}\left(v_{n}\right)<u_{n}(x)+\varepsilon,
$$

where $v_{n}=\left(\theta_{1}, \xi_{1}, v_{n-1}\right)$. This completes the proof of (4.1).

By construction, $u_{n} \searrow u$. Thus, by (4.1),

$$
u(x) \leq \inf _{v_{n}} J_{x}\left(v_{n}\right) \text { for all } n
$$

For $\varepsilon>0$, there exists a strategy $v_{m}$ such that 


$$
u(x) \leq J_{x}\left(v_{m}\right)<V(x)+\varepsilon .
$$

Thus $u \leq V$. To show $u \geq V$, there exists index $j$ large enough and strategy $v_{j}$, such that

$$
J_{x}\left(v_{j}\right)<u_{j}(x)+\varepsilon / 2<u(x)+\varepsilon
$$

and hence $V(x) \leq u(x)$.

Similar control representations can be obtained in the bounded domain case.

ACKNOWLEDGEMENT. The research was supported in part by NSF grant \#DMS8508651, Institute for Mathematics and Its Applications, Minneapolis, and University of Tennessee Science Alliance Research Incentive Award.

\section{REFERENCES}

.. Z:irS, in. H. s., Piecewise-determiniotic Merkov processes: A general class of non-diffunion modelo, J. Royal Statist. B. 46 (1984), 353-388.

2. DAVIS, M. H. A., Control of Piecewise-deterministic via Discrede-time Dymamic Programming, in "Proceedings of 3rd Bad Hannef Symposium on Stochastic Differential Systems," 1985.

3. VERMES, D., Optimal control of piecewise-determiniotic Markov processes, Stochastics 14 (1985), 165-208.

4. SONER, M., Optimal Control with State-Space Conotraint II, SIAM J. of Control and Optim. 24 (1986), 1110-1122.

5. LENHART, S. M. and LIAO, Y. C., Integro-differential equations associated with optimal atopping time of a piecewise-deterministic process, Stochastics 18 (1985), 183-207.

6. LENHART, S. M. and LIAO, Y. C., Switching Control of Piecewise-Deterministic Processes, Journal of Optim. Theory and Applications (to appear).

7. GUGerLI, U. S., Optimal Stopping of a Piecewise-Deterministic Markov Process, Stochastics 19 (1986), 221-236.

8. BARLES, G., Deterministic Impulsive Control Problems, SIAM J. of Control and Optimization ( ).

9. CRANDALL, M. G., EVANS, L. C., and LIONS, P. L., Some properties of viscosity solutzons of Hamilton-Jacobi equations, Trans. A. M. S. 282 (1984), 487-502.

10. CRANDALL, M. G. and LIONS, P. L., Viscosity Solutions of Hamilton-Jacobi Equations, Trans. A. M. S. 93 (1983), 1-44.

11. LIONS, P. L. and PERTHAME, B., Quasi-variational Inequalitses and Ergodic Impulse Control, SIAM J. of Control and Optimization 24 (1986), 604-615.

12. LENHART, S. M., Viscosity Solutions Associated with Switching Control Problems for Piecewise-Deterministic Processes, Houston J. of Math. (to appear).

13. HANOUZET, B. and JOLY, J. L., Convergence uniforme des itérés définnisant la solutzon d'une inéquation quasivariationelle abstraite, Compte Rendus (Paris) 286 A (1978), 735-745.

14. PERTHAME, B., Inequations Quasi-Variationnelles et Equations de Hamilton-Jacobi-Bellman dans $\mathbf{R}^{n}$, Ann. de Toulouse 5 (1983), 237-257.

15. PERTHAME, B., Quasivariational Inequalztres and Hamilton-Jacobi-Bellman Equations in a Bounded Region, Comm. P. D. E. 9 (1984), 561-595.

16. BARLES, G., Quasi-variational inequalitzes and first order Hamilton-Jacobi equations, Thesis, Universite de Paris IX-Dauphine (1984).

17. BENSOUSSAN, A. and LIONS, J.L.,, Impulse Control and Quasi-variational Inequalities, Dunod, Paris (1984). 


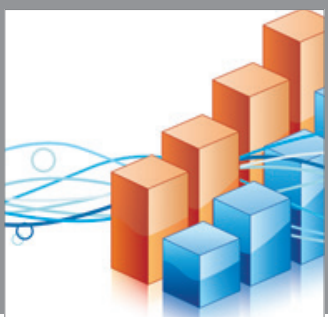

Advances in

Operations Research

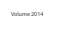

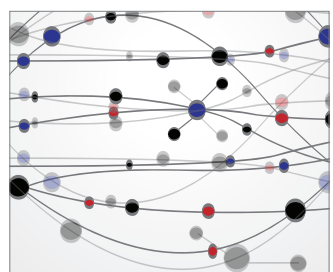

\section{The Scientific} World Journal
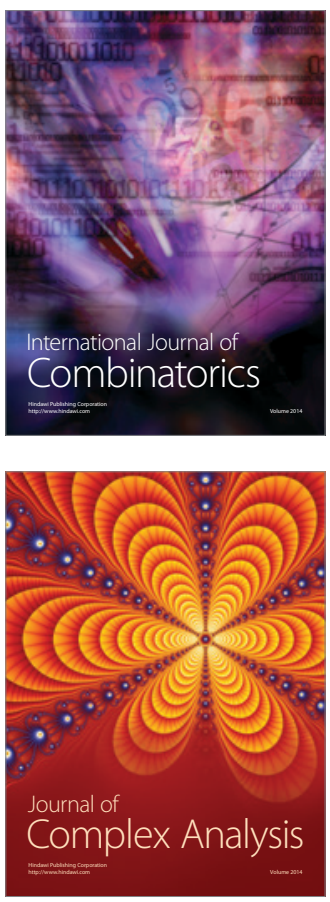

International Journal of

Mathematics and

Mathematical

Sciences
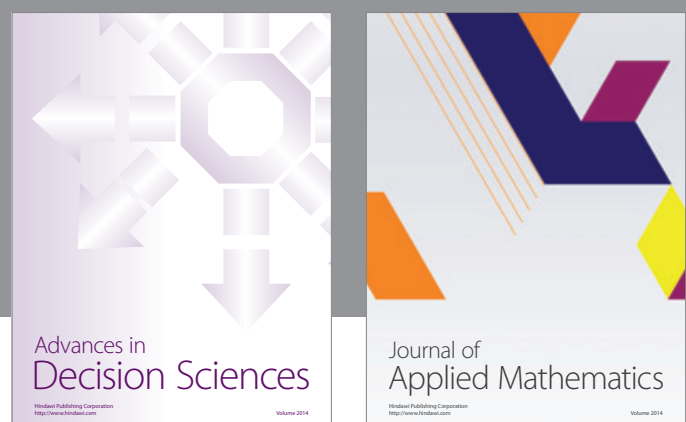

Journal of

Applied Mathematics
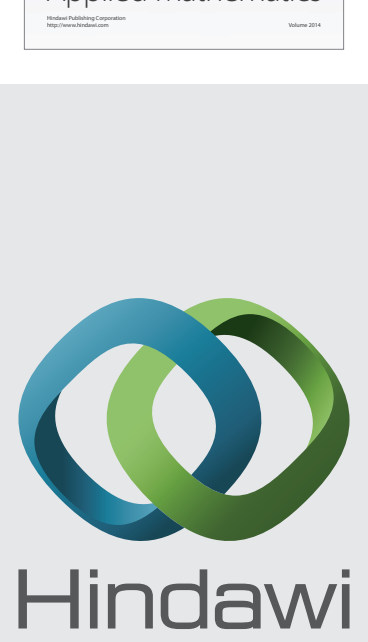

Submit your manuscripts at http://www.hindawi.com
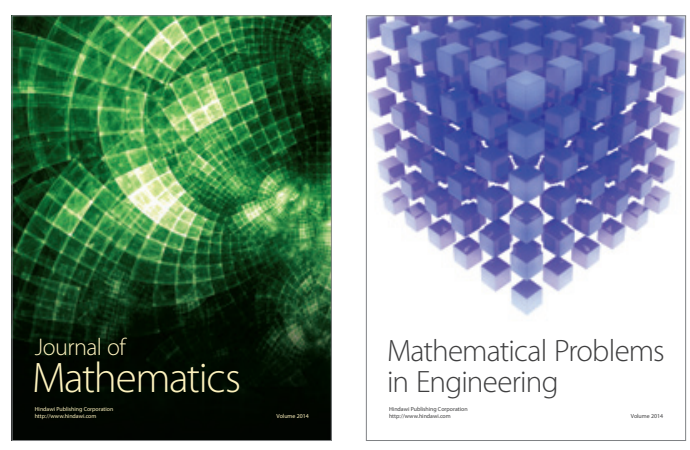

Mathematical Problems in Engineering
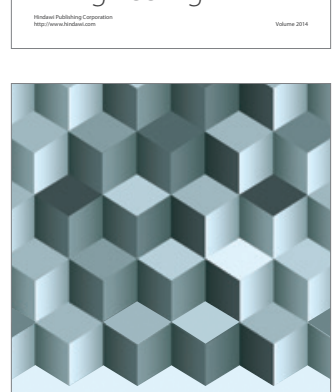

Journal of

Function Spaces
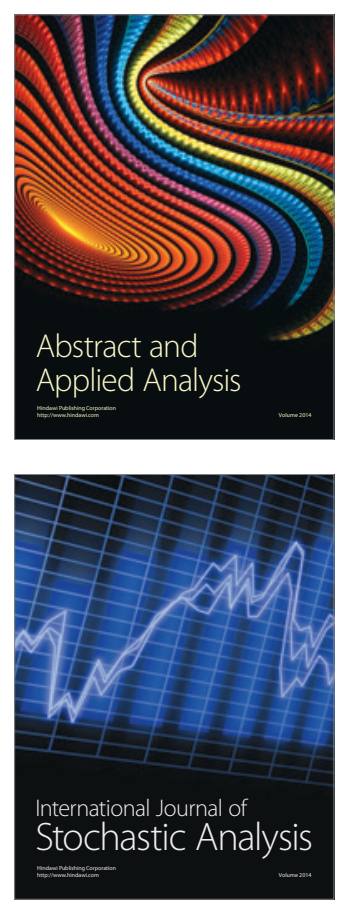

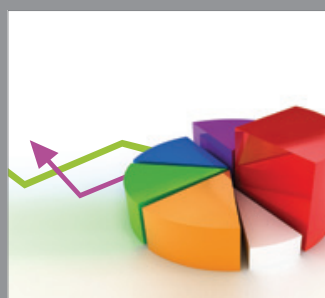

ournal of

Probability and Statistics

Promensencen
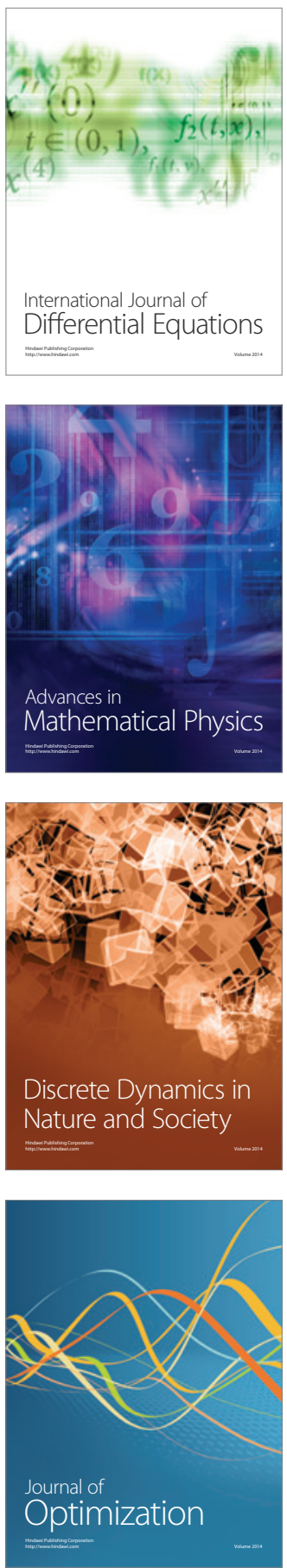